\title{
Detection of minority drug resistant mutations in Malawian HIV-1 subtype C-positive patients initiating and on first-line antiretroviral therapy
}

\begin{tabular}{|c|c|}
\hline \multicolumn{2}{|c|}{ Authors: } \\
\hline \multicolumn{2}{|c|}{ Zhiyong Zhou ${ }^{1}$ (D) } \\
\hline \multicolumn{2}{|c|}{ Kevin Tang ${ }^{2}$} \\
\hline \multicolumn{2}{|c|}{ Guoqing Zhang ${ }^{1}$} \\
\hline \multicolumn{2}{|c|}{ Nellie Wadonda-Kabondo ${ }^{3}$} \\
\hline \multicolumn{2}{|c|}{ Kundai $\mathrm{Moyo}^{3}$} \\
\hline \multicolumn{2}{|c|}{ Lori A. Rowe ${ }^{2}$} \\
\hline \multicolumn{2}{|c|}{ Joshua R. DeVos ${ }^{1}$} \\
\hline \multicolumn{2}{|c|}{ Nick Wagar ${ }^{1}$} \\
\hline \multicolumn{2}{|c|}{ Du-Ping Zheng ${ }^{1}$ (1) } \\
\hline \multicolumn{2}{|c|}{ Hongxiong $\mathrm{Guo}^{1}$} \\
\hline \multicolumn{2}{|c|}{ John Nkengasong $^{1}$} \\
\hline \multicolumn{2}{|c|}{ Mike Frace $^{2}$} \\
\hline \multicolumn{2}{|c|}{ Scott Sammons ${ }^{2}$} \\
\hline \multicolumn{2}{|c|}{ Chunfu Yang ${ }^{1}$} \\
\hline \multirow{8}{*}{\multicolumn{2}{|c|}{$\begin{array}{l}\text { Affiliations: } \\
{ }^{1} \text { International Laboratory } \\
\text { Branch, Division of Global } \\
\text { HIV \& TB, Center for Global } \\
\text { Health, Centers for Disease } \\
\text { Control and Prevention, } \\
\text { Atlanta, Georgia, } \\
\text { United States }\end{array}$}} \\
\hline & \\
\hline & \\
\hline & \\
\hline & \\
\hline & \\
\hline & \\
\hline & \\
\hline \multirow{5}{*}{\multicolumn{2}{|c|}{$\begin{array}{l}{ }^{2} \text { Biotechnology Core Facility } \\
\text { Branch, Division of Scientific } \\
\text { Resources, Centers for } \\
\text { Disease Control and } \\
\text { Prevention, Atlanta, Georgia, } \\
\text { United States }\end{array}$}} \\
\hline & \\
\hline & \\
\hline & \\
\hline & \\
\hline \multirow{3}{*}{\multicolumn{2}{|c|}{$\begin{array}{l}{ }^{3} \text { Department of Preventive } \\
\text { Health, Ministry of Health, } \\
\text { Lilongwe, Malawi }\end{array}$}} \\
\hline & \\
\hline & \\
\hline \multirow{2}{*}{\multicolumn{2}{|c|}{$\begin{array}{l}\text { Corresponding author: } \\
\text { Zhiyong Zhou, } \\
\text { zaz6@cdc.gov }\end{array}$}} \\
\hline & \\
\hline \multicolumn{2}{|l|}{ Dates: } \\
\hline \multicolumn{2}{|c|}{ Received: 13 Oct. 2017} \\
\hline \multicolumn{2}{|c|}{ Accepted: 01 Feb. 2018} \\
\hline \multicolumn{2}{|c|}{ Published: 30 May 2018} \\
\hline \multicolumn{2}{|l|}{ Read online: } \\
\hline 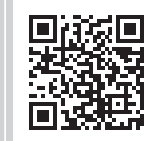 & $\begin{array}{l}\text { Scan this QR } \\
\text { code with your } \\
\text { smart phone or } \\
\text { mobile device } \\
\text { to read online. }\end{array}$ \\
\hline
\end{tabular}

Background: Minority drug resistance mutations (DRMs) that are often missed by Sanger sequencing are clinically significant, as they can cause virologic failure in individuals treated with antiretroviral therapy (ART) drugs.

Objective: This study aimed to estimate the prevalence of minor DRMs among patients enrolled in a Malawi HIV drug resistance monitoring survey at baseline and at one year after initiation of ART.

Methods: Forty-one plasma specimens collected from HIV-1 subtype C-positive patients and seven clonal control samples were analysed using ultra-deep sequencing technology.

Results: Deep sequencing identified all 72 DRMs detected by Sanger sequencing at the level of $\geq 20 \%$ and 79 additional minority DRMs at the level of $<20 \%$ from the 41 Malawian clinical specimens. Overall, DRMs were detected in $85 \%$ of pre-ART and $90.5 \%$ of virologic failure patients by deep sequencing. Among pre-ART patients, deep sequencing identified a statistically significant higher prevalence of DRMs to nucleoside reverse transcriptase inhibitors (NRTIs) compared with Sanger sequencing. The difference was mainly due to the high prevalence of minority K65R and M184I mutations. Most virologic failure patients harboured DRMs against both NRTIs and non-nucleoside reverse transcriptase inhibitors (NNRTIs). These minority DRMs contributed to the increased or enhanced virologic failures in these patients.

Conclusion: The results revealed the presence of minority DRMs to NRTIs and NNRTIs in specimens collected at baseline and virologic failure time points. These minority DRMs not only increased resistance levels to NRTIs and NNRTIs for the prescribed ART, but also expanded resistance to additional major first-line ART drugs. This study suggested that drug resistance testing that uses more sensitive technologies, is needed in this setting.

\section{Introduction}

Rapid scale-up of antiretroviral therapy (ART) over the past decade has remarkably reduced the mortality and morbidity of HIV-positive patients and decreased HIV transmission. Seventeen million HIV-1-positive patients around the world were receiving ART by the end of 2015. ${ }^{1}$ However, the scale-up of ART in resource-limited settings without adequate treatment monitoring has raised concern about the development of HIV drug resistance. The quasi-species nature of HIV-1 makes the detection of drug resistant mutations (DRMs) more difficult, because the commonly-used Sanger sequencing for drug resistance testing is incapable of detecting these drug resistant HIV variants at a level of less than $20 \%$ of the viral population. $2,3,4,5$

Minority drug resistant variants (also known as low-frequency mutants) that are not detected by Sanger sequencing are clinically important, as they can cause virologic failure in patients treated with ART for the first time. $6,7,8,9$ Recent studies have demonstrated that particular drug resistant HIV mutant viruses are clinically significant at a level of $1 \%$ of the viral population, as the minority variants can replicate quickly and become the predominant viral population through the selective pressure of ART drugs, leading to treatment failure. ${ }^{9,10}$ However, in the absence of drug pressure in treatment-naïve patients, the stability of transmitted DRMs is different. ${ }^{11}$ For instance, a transmitted M184V mutation can quickly revert to wild-type due to diminished viral fitness. ${ }^{12}$

How to cite this article: Zhou Z, Tang K, Zhang G, et al. Detection of minority drug resistant mutations in Malawian HIV-1 subtype C-positive patients initiating and on first-line antiretroviral therapy. Afr J Lab Med. 2018;7(1), a708. https://doi.org/10.4102/ajlm. v7i1.708

Copyright: @ 2018. The Authors. Licensee: AOSIS. This work is licensed under the Creative Commons Attribution License. 
In patients on ART, minority DRMs may persist for months or years during and post-ART. ${ }^{13,14,15}$ These minority DRMs, not detected by Sanger sequencing, present a need for more sensitive methods to detect the minority DRMs in a clinical sample.

Deep sequencing or next-generation sequencing technologies are extensively used to examine HIV viral diversity and minority drug resistant variants. Next-generation sequencing is a highly sensitive and high-throughput sequencing platform. It can detect HIV variants that make up $0.05 \%$ to $1 \%$ of viral populations. ${ }^{16,17,18,19,20,21}$

As part of HIV drug resistance surveillance by the Malawi Ministry of Health, a prospective cohort study to monitor ART outcomes and drug resistance development was conducted among patients from ART initiation to one year later. In this 2008 ART patient monitoring survey, $6.1 \%$ of the patients on ART for 12-15 months harboured drug resistant HIV. ${ }^{22}$ The most common non-nucleoside reverse transcriptase inhibitor (NNRTI) mutations were K103N (58.1\%), Y181C $(41.9 \%)$ and G190A (6.5\%), and the most frequent nucleoside reverse transcriptase inhibitor (NRTI) mutation was M184V (61.3\%). The DRMs conferring resistance against NNRTI at baseline were associated with DRMs detected at 12-15 months on ART. ${ }^{22}$ The present study aimed to evaluate parallel tagged deep sequencing primers on clinical samples and to investigate the profile of minority DRMs and their association with virologic failure in the same Malawi ART monitoring cohort.

\section{Methods}

\section{Ethical considerations}

The study protocol was approved by the National Health Sciences Research Committee of Malawi Institutional Review Board (\#1001). The use of de-identified data and drug resistance testing using Sanger sequencing and Roche 454 deep sequencing at the Centers for Disease Control and Prevention's (CDC) global HIV drug resistance laboratory, was determined to be non-human subjects research under CDC protocol \#6501 by the Office of the Associate Director for Science at the Center for Global Health, CDC, Atlanta, Georgia, United States.

\section{Clinical samples}

Between February and June 2008, HIV-1-positive patients aged 15 years or older, who initiated first-line ART at four ART clinics following the Malawi ART guidelines, were enrolled. Patients were treated with a first-line regimen combination of stavudine, lamivudine and nevirapine, or an alternative firstline regimen of stavudine to zidovudine substitutions in case of toxicity. Plasma specimens were collected before ART initiation and at 12-15 months on ART for viral load and HIV drug resistance testing. ${ }^{22}$ In the present study, we selected plasma specimens that had enough volume available to evaluate the assay. These were 20 samples collected from participants before ART initiation with viral loads ranging from 10471 to 2041738 copies/mL and 21 samples collected from ART patients at virologic failure (defined as viral load $\geq 1000$ copies $/ \mathrm{mL}$ ) after 12-15 months on ART (viral load ranging from 1738 to 776247 copies $/ \mathrm{mL}$ ). In addition, six plasmid clones and one mixed clone containing $1 \%$ mutant ( 2495 copies $/ \mu \mathrm{L}$ ) under the background of a wild-type clone were prepared and used to verify sequence accuracy in this study. All of these plasmid clones were derived from the Malawian cohort samples. All plasmids were constructed using $\mathrm{TOPO}^{\mathrm{TM}}$ vectors in Escherichia coli (Thermo Fisher Scientific, Carlsbad, California, United States). The six wildtype plasmid clones contained the HIV-1 pol gene without any DRMs, and the mutant clone contained DRMs at codons 103, 181, 184 and 190 of the HIV-1 pol gene.

\section{Viral ribonucleic acid extraction and reverse transcriptase polymerase chain reaction}

RNA was extracted from plasma specimens using the automated Abbott ${ }^{\mathrm{TM}}$ Sample Preparation System (m2000sp) (Abbott Laboratories. Abbott Park, Illinois, United States). The copy number of HIV-1 RNA was measured using RTqPCR on the m2000rt Real Time Analyzer (Abbott Laboratories. Abbott Park, Illinois, United States). ${ }^{22}$ The viral RNA was subjected to one-step RT-PCR amplification as described previously. ${ }^{11}$

\section{Parallel deep sequencing}

Degenerate primers, capable of amplifying multiple HIV-1 group $\mathrm{M}$ subtypes, were designed based on the HIV-1 pol gene sequences (www.hiv.lanl.gov) (Table 1). Six overlapping primer sets (forward and reverse) were used for bidirectional coverage of protease amino acids 6 to 99 and reverse transcriptase amino acids 1 to 251 . The size of the assembled gene fragment was 1035 base pairs. These six primers, tailed with Roche 454 adaptor and multiplex identifier sequences (tags), were synthesised at the CDC Biotechnology Core Facility. For PCR

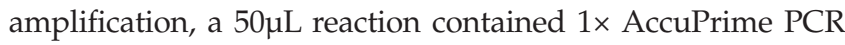
Buffer II (Thermo Fisher Scientific, Carlsbad, California, United States), 0.5 U AccuPrime Taq High Fidelity (Thermo Fisher Scientific, Carlsbad, California, United States), $13.5 \mu \mathrm{L}$ water, $0.3 \mu \mathrm{M}$, each forward and reverse primer, and $2 \mu \mathrm{L}$ DNA. All reactions were performed in 9700 thermal cyclers

TABLE 1: HIV-1 primers designed and used for amplifying pol gene amplicons with the deep sequencing method.

\begin{tabular}{lllc}
\hline $\begin{array}{l}\text { Amplicon } \\
\text { name }\end{array}$ & $\begin{array}{l}\text { Primer } \\
\text { name }\end{array}$ & Primer sequence & $\begin{array}{c}\text { HXB2 } \\
\text { position }\end{array}$ \\
\hline Amplicon 1 & PR1F & CTTTARCTTCCCTCARATCACTCT & $2243-2266$ \\
& PR1R & TCTTCCAATTATGTTGACAGG & $2513-2493$ \\
Amplicon 2 & PR2F & ATGGAAACCAARAATGATAG & $2375-2394$ \\
& PR2R & TTYTCTTCTGTYAATGGCCA & $2638-2619$ \\
Amplicon 3 & RT1F & AGTCCTATTGARACTGTRCCAGT & $2556-2578$ \\
& RT1R & CTGAAATCTACTAATTTYCTCCA & $2782-2760$ \\
Amplicon 4 & RT2F & AATTGGGCCTGAAATCCATAYAAIACTCC & $2696-2725$ \\
& RT2R & GGAATATTGCIGGTGATCCTTTCC & $3030-3007$ \\
Amplicon 5 & RT3F & ACAGTACTRGATGTGGGKGATGCATA & $2868-2893$ \\
& RT3R & TATTTCTAARTCAGATCCTACATA & $3134-3111$ \\
Amplicon 6 & RT4F & CAATATTCCARAGTAGCATGAC & $3022-3043$ \\
& RT4R & TTCTGTATRTCATTGACAGTCCA & $3325-3303$ \\
\hline
\end{tabular}


(Applied Biosystems, Austin, Texas, United States) under the following program: $95^{\circ} \mathrm{C}$ for 10 minutes (min); five cycles of $94^{\circ} \mathrm{C}$ for 20 seconds (s), $48^{\circ} \mathrm{C}$ for $20 \mathrm{~s}$, and $72^{\circ} \mathrm{C}$ for $1 \mathrm{~min}$ for annealing the primers with unique molecular tags followed by 35 cycles of $94^{\circ} \mathrm{C}$ for $30 \mathrm{~s}, 60^{\circ} \mathrm{C}$ for $20 \mathrm{~s}, 72^{\circ} \mathrm{C}$ for $30 \mathrm{~s}$; and one cycle of $72^{\circ} \mathrm{C}$ for $5 \mathrm{~min}$. The reaction products were confirmed by $1 \%$ agarose gel electrophoresis. The amplicons were purified using the Agencourt AMPure XP beads (Beckman Coulter, Beverly, Massachusetts, United States) or QIAGEN gel purification kits (QIAGEN, Germantown, Maryland, United States) and then quantified using the Quant-iT PicoGreen dsDNA kit (Thermo Fisher Scientific, Carlsbad, California, United States). Each sample had its own unique tag sequences for its six amplicons. Six barcoded samples were pooled and sequenced in one region. A total of eight regions were used for 48 samples on a plate. PCR amplicons from six samples were pooled in equal amounts, and amplified in water-in-oil emulsion PCR at the CDC Biotechnology Core Facility. Amplicons of 41 field samples and seven plasmid DNA samples were sequenced on the 454 platform (GS-FLX, Roche Applied Science, Indianapolis, Indiana, United States).

\section{Deep sequencing analysis}

Sequence files generated by Roche 454 deep sequencing were analysed using GS Amplicon Variant Analyzer pipeline from Roche Applied Science (Indianapolis, Indiana, United States). The deep sequencing analysis process in the present study included quality restriction for base call setting at 60 for signal intensity. All amplicon reads of alignment and single nucleotide polymorphism (SNP), calling against the HXB2 reference sequence, were evaluated with a quality score $\geq 25$ and read length $\geq 220$ base pairs. Sequence accuracy of Roche 454 runs was evaluated using the sequences generated by Sanger sequencing of the seven plasmid clones. The minority variant was defined as a SNP detected at $>0.68 \%$ and $<20 \%$ of the frequency of mutations. For DRM analysis, mutations were called and grouped based on International AIDS Society (IAS)-USA 2011 recommendations. ${ }^{23}$ Additional mutations for those samples collected before ART initiation were analysed based on the 2009 World Health Organization surveillance DRM list. ${ }^{24}$

\section{Standard Sanger sequencing}

The standard genotyping of HIV drug resistance was performed on all plasma samples using an in-house population-based sequencing assay. ${ }^{11}$ Raw sequencing data were analysed using the customised ReCall software, v.2.24 (provided by Dr. Richard Harrigan from British Columbia's Center for Excellence in HIV/AIDS Research, Vancouver, Canada). ${ }^{25}$ The mixed mutation calling threshold was set at $\geq 20 \%$ of the main peak. The DRMs were analysed as stated above for parallel deep sequencing results.

\section{Statistical analysis}

All statistical analysis was performed using SPSS Statistics v19 (SPSS Inc., Chicago, Illinois, United States). The Wilcoxon
Signed-Rank test was used to analyse the statistical differences in the number of DRMs detected between Sanger sequencing and deep sequencing. Fisher's exact test was used to compare the prevalence of HIV drug resistance by these two methods. A $P$-value of $<0.05$ was considered statistically significant.

\section{Results \\ Parallel deep sequencing coverage and estimate of sequence errors}

The GS-FLX deep sequencing of a single run yielded 246849 raw sequence reads, of which 242246 (98.1\%) reads passed the quality restrictions with a mean read length of 270 nucleotides, while 4603 (1.9\%) low-quality sequences were removed from the analysis pipeline. On average, 5490 reads per sample were obtained (range from 420 to 5673 reads). From the $1 \%$ mixed clone, $\mathrm{K} 103 \mathrm{~N}$ was not detected within the 520 sequence reads; Y181C and M184V were detected at the level of $1.03 \%$ with 1335 reads, while G190A was detected at $0.97 \%$ with 785 reads. The mean error rate plus two standard deviations was 0.43 from the six control plasmid clones. Sequence errors mostly occurred at the overlapping areas of amplicons in the RT gene. There was only one SNP showing an error rate of $0.68 \%$ at codon 17 of the $P R$ gene in a polyG region (GGGGGGCA, nucleotide 35, Figure 1). This error at $P R$ codon 17 was not in the DRM position according to IAS and Stanford HIV database definitions. Another high-error site was codons 63 of the RT gene (nucleotides 470 and 471, Figure 1), with a $0.61 \%$ error rate. Based on these background errors for each nucleotide position, the frequency $>0.68 \%$ error rate was used as the threshold for true SNPs when evaluating the minority DRMs in the clinical samples.

\section{Comparative analysis of drug resistance mutations detected by parallel deep sequencing and Sanger sequencing}

Barcoded deep sequencing primers amplified all amplicons from the clinical samples. Roche GS-FLX deep sequencing identified all 72 of the DRMs that had been detected by Sanger sequencing at a level of $\geq 20 \%$ from the 41 clinical Malawian samples. Additionally, a total of 79 DRMs were exclusively detected by deep sequencing. Thus, Sanger sequencing missed $52.3 \%$ of DRMs at a level of $<20 \%$ in these clinical samples. The differences in the numbers of DRMs detected by these two sequencing approaches were significant against NRTIs $(p=0.004)$ and NNRTIs $(p=0.0001)$. Further, the Cohen's effect size value $(d=0.91$ and 0.79$)$ suggests a moderate to high practical significance. No significant difference was found in detection of protease inhibitor (PI) mutations $(p=0.083)$ (Figure 2).

\section{Prevalence of minority drug resistance mutations in baseline samples before antiretroviral therapy initiation}

Overall, the frequency of DRMs increased by deep sequencing with minority DRMs being detected in 17 $(85 \%)$ of the 20 pre-ART samples by deep sequencing. 


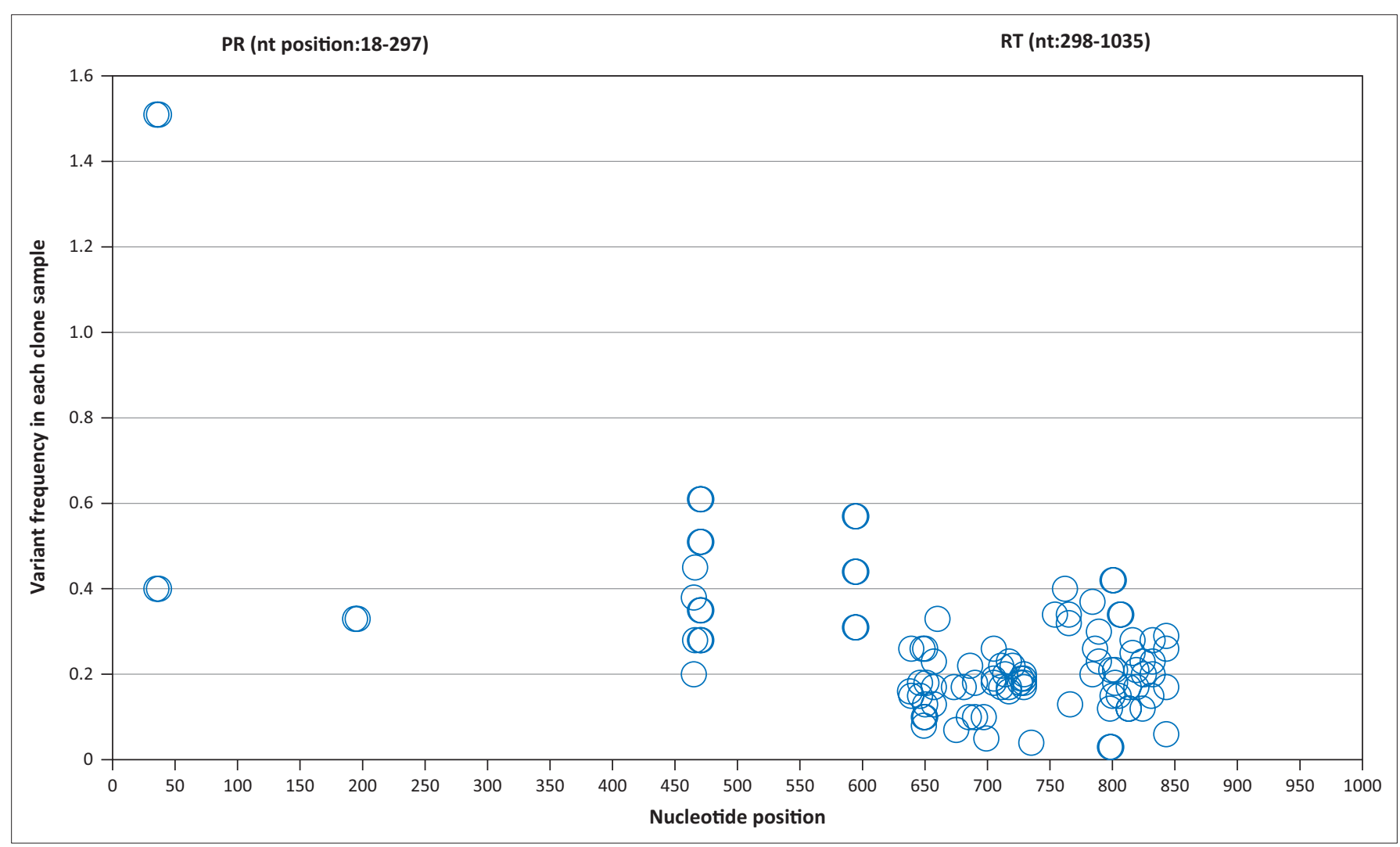

nt, nucleotide; PR, protease; RT, reverse transcriptase.

FIGURE 1: Error frequency of 454 deep sequencing on the six plasmid control clones at each nucleotide position from amplified amplicons. The $X$ axis is the nucleotide sequence location in protease and reverse transcriptase genes; the $\mathrm{Y}$ axis is the percentage of single nucleotide polymorphism error.

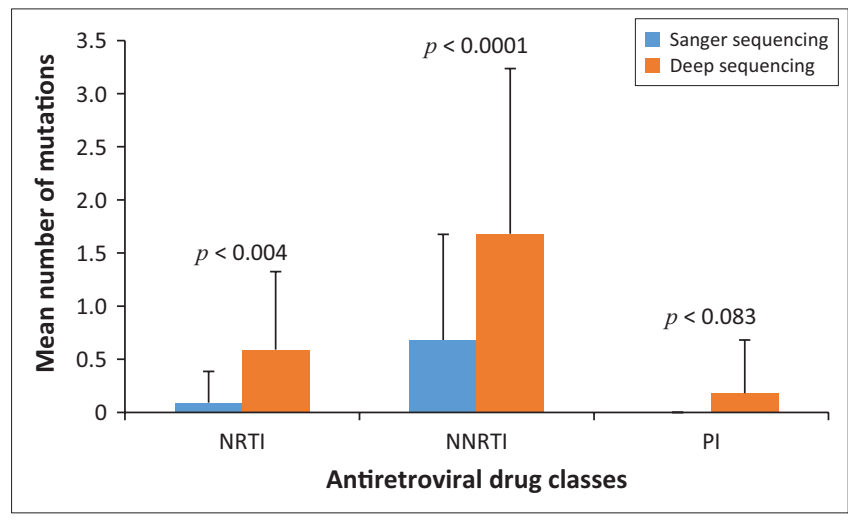

NRTI, nucleoside reverse transcriptase inhibitors; NNRTI, non-nucleoside reverse transcriptase inhibitors; PI, protease inhibitor.

FIGURE 2: Comparison of mean number of HIV-1 drug resistant mutations detected by Sanger and deep sequencing methods. The vertical bars present mean number of mutations \pm standard error; $p<0.05$ was considered statistically significant.

Minority NRTI were detected in $10(50 \%)$ of these samples and NNRTI mutations in $14(70 \%)$, while minority PI mutations were found in three $(15 \%)$ of the 20 samples. In addition, the number of DRMs detected by deep sequencing was higher than Sanger sequencing. This increase of DRM numbers detected by deep sequencing was statistically significant for NRTI (2/20 vs $13 / 20, p=0.015)$, but not for NNRTI $(9 / 20$ vs $14 / 20, p=0.13)$ or PI $(0 / 20$ vs $3 / 20, p=$ $0.235)$ when compared to Sanger sequencing. The most common minority NRTI mutation was K65R (6 of 20), followed by M184I (2 of 20, Table 2). The SNP frequency for
K65R ranged from $0.7 \%$ to $2.5 \%$ of the sequence reads in these six patients and both mutant alleles of AGG and AGA were detected in these HIV-1 subtype C-positive samples. The common minority NNRTI mutations detected were V106M, V179T and G190A (3/20 each), followed by E138K and Y181C (2/20 each), and K101P, K103N, V108I, E138A, V179D, A190E and H221Y (1/20 each). The minority PI mutations detected in three of the samples were M46I $(2 / 20)$ and D30N (1/20) (Table 2$)$.

\section{Prevalence of minority drug resistance mutations in patients experiencing virologic failure after antiretroviral therapy}

Overall, minority DRMs were detected by deep sequencing in $90.5 \%(19 / 21)$ of the patients failing ART. Of the 21 Malawian samples, minority NRTI mutations were detected in $8(38 \%)$ samples, while minority NNRTI mutations were detected in $14(66.7 \%)$ samples (Table 2). Minority PI mutations were only detected in 3 of $21(14.3 \%)$ samples. However, the increased number of minority mutations in individual patients for NRTI or NNRTI in ART-failing patients was not statistically significant (NRTI: $12 / 21$ vs $14 / 21, p>0.05$; or NNRTI: $18 / 21$ vs $19 / 21, p>0.05)$ when compared to Sanger sequencing. Among the minority NRTI mutations, M184I was detected in three samples, and $\mathrm{M} 184 \mathrm{~V}$ and $\mathrm{K} 65 \mathrm{R}$ in two samples respectively. Other minority mutations including M41L, A62V, D67N, T69D, V75M, T215F and K219E were found in one sample each. Among the minority NNRTI mutations, V108I, V179T were 
TABLE 2: Number of drug resistance mutations detected by two sequencing methods against PIs, NRTIs and NNRTIs from plasma specimens collected from patients before ART initiation and at virologic failure $\dagger$

\begin{tabular}{|c|c|c|c|c|c|c|c|}
\hline \multirow[t]{2}{*}{ Drug class } & \multirow[t]{2}{*}{ Mutation } & \multicolumn{3}{|c|}{ Before ART initiation $(n=20)$} & \multicolumn{3}{|c|}{ At virologic failure $(n=21)$} \\
\hline & & $\begin{array}{l}\text { No. of mutations } \\
\text { detected by Sanger }\end{array}$ & $\begin{array}{l}\text { No. of mutations } \\
\text { detected by NGS }\end{array}$ & $\begin{array}{c}\text { No. of mutations } \\
\text { detected by NGS only }\end{array}$ & $\begin{array}{l}\text { No. of mutations } \\
\text { detected by Sanger }\end{array}$ & $\begin{array}{l}\text { No. of mutations } \\
\text { detected by NGS }\end{array}$ & $\begin{array}{c}\text { No. of mutations } \\
\text { detected by NGS only }\end{array}$ \\
\hline \multirow[t]{3}{*}{$\mathrm{PI}$} & D30N & 0 & 1 & 1 & 0 & 0 & 0 \\
\hline & M46I & 0 & 2 & 2 & 0 & 2 & 2 \\
\hline & M46L & 0 & 0 & 0 & 0 & 1 & 1 \\
\hline Subtotal & & 0 & 3 & 3 & 0 & 3 & 3 \\
\hline \multirow[t]{12}{*}{ NRTI } & M41L & 0 & 0 & 0 & 0 & 1 & 1 \\
\hline & E44D & 1 & 1 & 0 & 1 & 1 & 0 \\
\hline & A62V & 0 & 0 & 0 & 2 & 3 & 1 \\
\hline & K65R & 0 & 6 & 6 & 2 & 4 & 2 \\
\hline & D67N & 0 & 0 & 0 & 3 & 4 & 1 \\
\hline & T69D & 0 & 0 & 0 & 0 & 1 & 1 \\
\hline & K70R & 1 & 1 & 0 & 0 & 0 & 0 \\
\hline & V75M & 0 & 0 & 0 & 0 & 1 & 1 \\
\hline & M184I & 0 & 2 & 2 & 0 & 3 & 3 \\
\hline & M184V & 0 & 0 & 0 & 11 & 13 & 2 \\
\hline & $\mathrm{T} 215 \mathrm{~F}$ & 0 & 0 & 0 & 0 & 1 & 1 \\
\hline & K219E & 0 & 0 & 0 & 0 & 1 & 1 \\
\hline Subtotal & & 3 & 11 & 8 & 21 & 35 & 14 \\
\hline \multirow[t]{18}{*}{ NNRTI } & V90I & 1 & 1 & 0 & 0 & 2 & 2 \\
\hline & A98G & 0 & 0 & 0 & 1 & 1 & 0 \\
\hline & K101E & 2 & 2 & 0 & 1 & 4 & 3 \\
\hline & K101P & 0 & 1 & 1 & 0 & 0 & 0 \\
\hline & K103N & 2 & 3 & 1 & 9 & 11 & 2 \\
\hline & V106A & 0 & 0 & 0 & 0 & 1 & 1 \\
\hline & V106M & 0 & 3 & 3 & 1 & 2 & 1 \\
\hline & V108I & 0 & 1 & 1 & 3 & 7 & 4 \\
\hline & E138A & 2 & 3 & 1 & 2 & 4 & 2 \\
\hline & V179E & 0 & 0 & 0 & 0 & 1 & 1 \\
\hline & V179T & 1 & 4 & 3 & 0 & 4 & 4 \\
\hline & Y181C & 5 & 7 & 2 & 9 & 10 & 1 \\
\hline & Y188C & 0 & 0 & 0 & 0 & 2 & 2 \\
\hline & Y188L & 0 & 0 & 0 & 0 & 1 & 1 \\
\hline & G190A & 1 & 4 & 3 & 1 & 4 & 3 \\
\hline & G190E & 0 & 1 & 1 & 0 & 1 & 1 \\
\hline & G190R & 0 & 0 & 0 & 1 & 1 & 0 \\
\hline & $\mathrm{H} 221 \mathrm{Y}$ & 1 & 2 & 1 & 4 & 7 & 3 \\
\hline Subtotal & & 15 & 35 & 20 & 33 & 64 & 31 \\
\hline
\end{tabular}

ART, antiretroviral therapy; NGS, next-generation sequencing; PI, protease inhibitor; NRTI, nucleoside reverse transcriptase inhibitor; NNRTI, non-nucleoside reverse transcriptase inhibitor. †Virologic failure was defined as plasma viral load $\geq 1000$ copies/mL after $12-15$ months on ART.

detected in four samples and G190A, H221Y and K101E were detected in three samples. Other NNRTI mutations, such as V90I, K103N, V106A/M, E138A, V179E, Y181C, $\mathrm{Y} 188 \mathrm{C} / \mathrm{L}$ and G190E were also found in one or two samples.

\section{Clinical impact of minority drug resistance mutations detected by deep sequencing on virologic failure}

To study the impact of minority DRMs detected by deep sequencing on the clinical outcome of patients on ART, we compared drug resistance levels (genotype susceptibility score) or expansion of drug resistance to additional drugs or drug classes from those 21 ART-failure patients against NRTIs and NNRTIs using the Stanford HIV drug resistance database tool. Among 19 patients with the additional minority DRMs detected, we found that seven (36.8\%) of the patients had enhanced resistance levels to NRTIs. More importantly, three of the seven patients had gained lowto high-level resistance against tenofovir (Table 3), a key component of the current World Health Organizationrecommended first- and second-line regimens. ${ }^{26}$ Fourteen (73.7\%) out of the 19 patients also had an enhanced resistance level to NNRTIs and 13 of these 14 patients had intensified or expanded resistance profiles against the second generation of NNRTIs (etravirine and rilpivirine) (Table 4). Furthermore, the drug resistance profile analyses revealed an intermediateor high-level resistance to the relevant first-line regimens (stavudine, lamivudine and nevirapine or zidovudine, lamivudine and nevirapine) that are prescribed to these Malawian patients and which might explain the virologic failures these patients experienced. 
TABLE 3: Comparison of NRTI drug resistance mutations detected from plasma specimens collected from patients with virologic failure after $12-15$ months on ART by Sanger sequencing and deep sequencing and their impacts on NRTI susceptibility.

\begin{tabular}{|c|c|c|c|c|}
\hline Sample ID & Mutations by Sanger & Drug resistance level (score) $\dagger$ & Mutations by deep sequencing & Drug resistance level (score) \\
\hline 5426 & D67N, M184V & $3 \mathrm{TC}(60), \mathrm{ABC}(15), \mathrm{DDI}(15), \mathrm{FTC}(60)$ & K65R,D67N, M184V & $\begin{array}{l}3 T C(90), A B C(65), D 4 T(60), D D I(75), \\
\text { FTC(90),TDF(65) }\end{array}$ \\
\hline 5471 & $\mathrm{~A} 62 \mathrm{~V}, \mathrm{~K} 65 \mathrm{R}, \mathrm{D} 67 \mathrm{~N}, \mathrm{M} 184 \mathrm{~V}$ & $\begin{array}{l}3 \mathrm{TC}(95), \mathrm{ABC}(70), \mathrm{D} 4 \mathrm{~T}(65), \mathrm{DDI}(80) \text {, } \\
\text { FTC(95),TDF(80) }\end{array}$ & M41L,K219E,A62V,K65R,D67N,M184V & $\begin{array}{l}3 T C(100), A B C(85), D 4 T(90), D D I(95), \\
\text { FTC(100),TDF(95),AZT(20) }\end{array}$ \\
\hline 5496 & $\mathrm{~A} 62 \mathrm{~V}, \mathrm{~V} 75 \mathrm{I}, \mathrm{M} 184 \mathrm{~V}$ & $3 \mathrm{TC}(70), \mathrm{ABC}(25), \mathrm{DDI}(20), \mathrm{FTC}(70)$ & A62V,V75I,M184V,T69D,T215F & $\begin{array}{l}3 T C(75), A B C(40), A Z T(45), D 4 T(55), D D I(65), \\
\text { FTC(75),TDF(15) }\end{array}$ \\
\hline 5506 & M184V & $3 \mathrm{TC}(60), \mathrm{ABC}(15), \mathrm{DDI}(10), \mathrm{FTC}(60)$ & M184V & $3 \mathrm{TC}(60), \mathrm{ABC}(15), \mathrm{DDI}(10), \mathrm{FTC}(60)$ \\
\hline 5549 & M184V & $3 \mathrm{TC}(60), \mathrm{ABC}(15), \mathrm{DDI}(10), \mathrm{FTC}(60)$ & D67N,M184V & $3 \mathrm{TC}(60), A B C(20), D D I(15), \mathrm{FTC}(60)$ \\
\hline 5642 & M184V & 3TC(60),ABC(15),DDI(10),FTC(60) & M184I,M184V & $3 \mathrm{TC}(60), \mathrm{ABC}(15), \mathrm{DDI}(10), \mathrm{FTC}(60)$ \\
\hline 5727 & M184V & $3 \mathrm{TC}(60), \mathrm{ABC}(15), \mathrm{DDI}(10), \mathrm{FTC}(60)$ & K65R,D67N,V75M,M184I,M184V & $\begin{array}{l}3 T C(90), A B C(65), D 4 T(100), D D I(90), \\
F T C(90), T D F(65)\end{array}$ \\
\hline 6525 & E44D,T69N,M184V & $3 \mathrm{TC}(60), \mathrm{ABC}(15), \mathrm{DDI}(20), \mathrm{FTC}(60)$ & E44D,T69N,M184V & $3 \mathrm{TC}(60), \mathrm{ABC}(15), \mathrm{DDI}(20), \mathrm{FTC}(60)$ \\
\hline 5713 & V118I, M184V & $3 \mathrm{TC}(60), \mathrm{ABC}(15), \mathrm{DDI}(10), \mathrm{FTC}(60)$ & A62V,V118I,M184V & $3 T C(65), A B C(20), D D I(15), F T C(65)$ \\
\hline 5597 & None & $\mathrm{S}$ & M184V & $3 T C(60), A B C(15), D D I(10), F T C(60)$ \\
\hline 5527 & M184V & $3 \mathrm{TC}(60), \mathrm{ABC}(15), \mathrm{DDI}(10), \mathrm{FTC}(60)$ & M184V & $3 \mathrm{TC}(60), \mathrm{ABC}(15), \mathrm{DDI}(10), \mathrm{FTC}(60)$ \\
\hline 5508 & K65R, D67N & $\begin{array}{l}3 \mathrm{TC}(30), \mathrm{ABC}(50), \mathrm{D} 4 \mathrm{~T}(60), \mathrm{DDI}(65), \\
\mathrm{FTC}(30), \mathrm{TDF}(65)\end{array}$ & $\mathrm{K} 65 \mathrm{R}, \mathrm{D} 67 \mathrm{~N}$ & $\begin{array}{l}3 \mathrm{TC}(30), \mathrm{ABC}(50), \mathrm{D} 4 \mathrm{~T}(60), \mathrm{DDI}(65), \mathrm{FTC}(30) \text {, } \\
\operatorname{TDF}(65)\end{array}$ \\
\hline 5715 & M184V & $3 \mathrm{TC}(60), \mathrm{ABC}(15), \mathrm{DDI}(10), \mathrm{FTC}(60)$ & M184V & $3 \mathrm{TC}(60), \mathrm{ABC}(15), \mathrm{DDI}(10), \mathrm{FTC}(60)$ \\
\hline 5434 & None & $\mathrm{S}$ & M184I,M184V & $3 T C(60), A B C(15), D D I(10), F T C(60)$ \\
\hline
\end{tabular}

ABC, abacavir; AZT, zidovudine; DDI, didanosine; FTC, emtricitabine; 3TC, lamivudine; TDF, tenofovir; $d 4 T$, stavudine.

$\dagger$, Scores 10-14: potential low-level of resistance; $15-29$, low-level of resistance; 30-59, intermediate-level of resistance; $\geq 60$, high-level of resistance;

$\mathrm{S}$, susceptible (0-9). The letters and numbers in italics indicate increased/expanded levels of drug resistance caused by minority drug resistance mutations detected by deep sequencing only

TABLE 4: Comparison of NNRTI drug resistance mutations detected from plasma specimens collected from patients with virologic failure after $12-15$ months on ART by Sanger sequencing and deep sequencing and their impacts on NNRTI susceptibility.

\begin{tabular}{|c|c|c|c|c|}
\hline Sample ID & Mutations by Sanger & Drug resistance level (score) $\dagger$ & Mutations by NGS & Drug resistance level (score) \\
\hline 5426 & Y181C,H221Y & $\operatorname{EFV}(40), \operatorname{ETR}(40), N V P(70), \operatorname{RPV}(40)$ & Y181C,H221Y & $\operatorname{EFV}(40), \operatorname{ETR}(40), N V P(70), \operatorname{RPV}(40)$ \\
\hline 5471 & Y106M,Y181C & $\operatorname{EFV}(90), N V P(120), E T R(30), R P V(30)$ & Y106M,Y181C & $\operatorname{EFV}(90), N V P(120), \operatorname{ETR}(30), \operatorname{RPV}(30)$ \\
\hline 5496 & $\mathrm{~K} 103 \mathrm{~N}$ & $\operatorname{EFV}(60), N V P(60)$ & V108I,K103N,E138A,H221Y, & $\operatorname{EFV}(80), N V P(85), E T R(20), R P V(25)$ \\
\hline 5506 & K103N,Y181C,G190A,H221Y & $\operatorname{EFV}(145), E T R(65), N V P(190), R P V(65)$ & K101E,K103N,V108I,Y181C,G190A,H221Y & $\operatorname{EFV}(170), E T R(80), N V P(235), R P V(95)$ \\
\hline 5549 & Y181C,G190R & $\operatorname{EFV}(30), \operatorname{ETR}(30), \operatorname{NVP}(60), \operatorname{RPV}(30)$ & V179E,Y181C,Y188C G190R & $\operatorname{EFV}(100), \operatorname{ETR}(40), N V P(130), R P V(40)$ \\
\hline 5642 & K103N & $\operatorname{EFV}(60), N V P(60)$ & K103N & $\operatorname{EFV}(60), N V P(60)$ \\
\hline 5727 & $\mathrm{~K} 103 \mathrm{~N}, \mathrm{Y} 181 \mathrm{C}, \mathrm{H} 221 \mathrm{Y}$ & $\operatorname{EFV}(100), \operatorname{ETR}(40), N V P(130), \operatorname{RPV}(40)$ & V90I,K103N,V179T,K138T,Y181C,H221Y & $\operatorname{EFV}(110), \operatorname{ETR}(50), N V P(140), R P V(50)$ \\
\hline 6525 & K101E,E138A,Y181I & $\operatorname{EFV}(45), \operatorname{ETR}(85), N V P(90), \operatorname{RPV}(105)$ & K101E,E138A,V179T,Y181I,H221Y & $\operatorname{EFV}(65), \operatorname{ETR}(105), N V P(110), R P V(125)$ \\
\hline 5713 & Y108I,Y181C,H221Y & $\operatorname{EFV}(50), E T R(40), N V P(85), R P V(40)$ & Y108I,V179T,Y181C,H221Y & $\operatorname{EFV}(60), \operatorname{ETR}(50), N V P(95), \operatorname{RPV}(50)$ \\
\hline 5585 & V179D & $\operatorname{EFV}(10), \operatorname{ETR}(10), N V P(10), \operatorname{RPV}(10)$ & V179D & $\operatorname{EFV}(10), \operatorname{ETR}(10), N V P(10), \operatorname{RPV}(10)$ \\
\hline 5597 & K103N & $\operatorname{EFV}(60), N V P(60)$ & K103N,V108I,E138A & $\operatorname{EFV}(70), N V P(75), R P V(15)$ \\
\hline 5778 & None & S & G190E & $\operatorname{EFV}(60), N V P(60), E T R(45), R P V(45)$ \\
\hline 5527 & A98G,Y181C & $\operatorname{EFV}(40), \operatorname{ETR}(40), N V P(90), \operatorname{RPV}(45)$ & A98G,K101E,V108I,V179T,Y181C,H221Y & $\operatorname{EFV}(85), E T R(75), N V P(155), R P V(95)$ \\
\hline 5665 & E138A & RPV(15) & K103N,E138A & $\operatorname{EFV}(60), N V P(60), \operatorname{RPV}(15)$ \\
\hline 5608 & K103N & $\operatorname{EFV}(60), N V P(60)$ & K103N,G190A & $\operatorname{EFV}(105), N V P(120), E T R(15), R P V(15)$ \\
\hline 5508 & K103N,Y108I & $\mathrm{EFV}(70), \mathrm{NVP}(75)$ & V90I,K103N,Y108I,Y181C,Y188L & $\operatorname{EFV}(160), N V P(195), E T R(45), R P V(90)$ \\
\hline 5715 & K103N,V108I & $\mathrm{EFV}(70), \mathrm{NVP}(75)$ & K101E,K103N,V108I,G190A & $\operatorname{EFV}(130), N V P(165), E T R(30), R P V(35)$ \\
\hline 5434 & K103N,Y181C & $\operatorname{EFV}(90), \operatorname{ETR}(30), N V P(120)$ & K103N,Y181C & $\operatorname{EFV}(90), \operatorname{ETR}(30), N V P(120)$ \\
\hline
\end{tabular}

ETR, etravirine; EFV, efavirenz; NVP, nevirapine; NGS, next-generation sequencing; RPV, rilpivirine.

$\uparrow$, Score 10-14: potential low-level of resistance; $15-29$, low-level of resistance; $30-59$, intermediate-level of resistance; $\geq 60$, high-level of resistance; S, susceptible. The letters and numbers in italics indicate increased/expanded levels of drug resistance caused by minority drug resistance mutations detected by deep sequencing only.

\section{Discussion}

The present study has shown that massively parallel deep sequencing is capable of detecting minority HIV-1 variants from HIV-1 subtype $\mathrm{C}$ clinical samples. The higher prevalence of minority DRMs in pre-ART Malawian patients to NRTIs by deep sequencing was statistically significant compared to Sanger sequencing. The minority mutation profile revealed that the increased minority DRMs were associated with enhanced DR levels in virologically failing patients.

This study was designed to evaluate parallel tagged deep sequencing in detection of minority DRMs in samples collected from Malawi. We successfully amplified all 41 plasma specimens collected from the patients and seven plasmid DNA samples that generated 242246 sequencing reads using the degenerate primers designed for HIV-1 group $\mathrm{M}$ subtypes and circulating recombinant forms. Our results not only showed $100 \%$ concordance of DRMs detected by Sanger sequencing and deep sequencing, but deep sequencing also detected over $50 \%$ minority DRMs in these clinical samples. At the lower detection level of $0.68 \%$, set by the current study, minority DRMs were detected in a majority of samples collected from patients before ART initiation and at virologic failure. Although we only analysed HIV-1 subtype C-positive Malawian samples in this study, the primers were designed for all relevant HIV-1 group $M$ subtypes and circulating recombinant forms. In fact, we were able to 
amplify subtype B, B/C, F and G samples using these primers which were confirmed by gel electrophoresis and successfully detect 5 of 5 subtype B samples using deep sequencing methods. ${ }^{27}$

Previous studies reported the use of parallel tagged deep sequencing methods in detecting low levels of HIV-1 subtype B variants. ${ }^{18,20,21,28,29}$ A study by Dudley et al. ${ }^{20}$ evaluated a 454 GS Junior sequencer by multiplexing 48 samples collected from HIV-1 subtype B-positive individuals and obtained a sequencing success rate of $93 \%$ and an error rate of $0.71 \%$. Our study using GS-FLX with multiplexing on 48 samples collected from HIV-1 subtype C-positive patients showed a $100 \%$ amplification rate and $0.265 \%$ mean error rate. Many studies have reported that the mean error rate of pyrosequencing techniques can be down to $0.05 \%$ to $1 \% .17,19,21,30$ The error rates for deep sequencing not only affect the accuracy of base calling, but also impact the sensitivity of minority variant detection. It has been reported that factors, such as the input number of template molecules, sequence primers, amplicon length, nucleotide sequences, PCR errors and operational procedures, might contribute to deep sequencing assay sensitivity and accuracy. ${ }^{17,31,32,33}$ In addition, error rates are nucleotide position-dependent and Roche 454 deep sequencing methods are prone to have more errors at the homopolymeric regions. ${ }^{17,31}$ In the present study, cross-over errors of major DRMs between samples were not found. To balance the detection sensitivity with detection accuracy, we set up the base calling threshold for low-frequency mutations at $>0.68 \%$ (mean error rate +2 standard deviations) which was calculated based on the error rates of individual nucleotide positions of six control plasmid samples. In the present study, the K103N mutation was not detected from the mixed clone at $1 \%$ of minority variant level of control plasmids from 520 reads. This was likely due to not having enough reads amplified for this mixed plasmid as a previous study demonstrated that at least 1950 reads are required for detecting a minority variant for $\mathrm{K} 103 \mathrm{~N}$ mutations at about the $1 \%$ level. ${ }^{17}$ One limitation of our study was that the primer pair for amplifying amplicons containing codon 103 of RT gene was not optimal for the depth of coverage. Some minority K103N mutations could have been missed in this study. Thus, the number of sequence reads obtained for each nucleotide position and errors at the homopolymeric regions played an important role in the depth of the next-generation sequencing.

Our results from samples collected from patients failing ART and initiating ART demonstrate that deep sequencing has the added benefit of detecting low-frequency mutations in this Malawian cohort. Overall, deep sequencing detected significantly more DRMs than Sanger sequencing. Of those specimens collected from patients initiating ART, more DRMs against NRTI were detected by deep sequencing. Among the minority DRMs, detected by 454 deep sequencing, K65R and M184I were the most common and may compromise the effectiveness of both first- and secondline drugs used according to the Malawi ART guidelines.
The K65R mutation can confer resistance to stavudine and cross-resistance to lamivudine, abacavir, emtricitabine and tenofovir, ${ }^{23,34,35}$ and is more frequently identified in HIV-1 subtype C. ${ }^{30,35,36}$ Similar to previous studies, the K65R mutation was seen in both treatment-naïve patients and patients failing ART in this cohort. Several studies have reported that increased presence of $\mathrm{K} 65 \mathrm{R}$ mutations is caused by pyrosequencing errors or by the nucleotide template of subtype $C$ viruses (such as the ATA sequence at codon 63 of the RT gene)..$^{30,32,37}$ Even though no errors at codon 65 of the RT gene were found by deep sequencing in the current study, we did find a relatively higher error rate at codon 63 of the $R T$ gene in the subtype C plasmid sequences. However, we did not find higher error rates for K65R compared with other DRM sites in these patients. The M184I mutations were only detected at low frequencies by deep sequencing in pre-ART patients and patients with treatment failure. M184I was considered to be a transient mutation before being replaced by M184V..$^{19,38,39}$ No detectable levels of M184V mutations were found in preART samples using deep sequencing, but M184V mutation was detected in over three-quarters of samples from patients failing ART. Taken together, the higher NRTI resistance mutations of M184V and K65R in patients failing ART were more likely acquired by selective drug pressure in this Malawian cohort treated with a regimen containing stavudine and lamivudine..$^{22}$

In this study, most samples collected from virologic failure patients had detectable DRMs to NNRTI by both Sanger sequencing and deep sequencing. The mutations K101E, K103N, V106A/M, V179D/T, Y181C, G190A/E and H221Y to NNRTI were the most common minority mutations detected in these patients. Virus with K101E/Y181C/G190A and other mutations could increase levels of resistance to nevirapine 893 -fold. ${ }^{40}$ The H221Y mutation could also impact clinical outcomes as Y181C/H221Y along with the K103N or K101Q mutations could increase resistance levels to nevirapine over 100-fold (K103N) or 3000-fold (K101Q). ${ }^{41}$ The drug resistance profile generated by deep sequencing revealed that these mutations were associated with the firstline regimen (stavudine, lamivudine and nevirapine). The DRMs to NNRTI in pre-ART samples were also relatively high in these patients and were likely due to single-dose nevirapine used in the prevention of mother-to-child transmission program in Malawi. ${ }^{22}$ However, our results could not rule out the presence of transmitted drug resistance to NNRTI in these pre-ART patients.

Although DRMs against PIs were not detected using Sanger sequencing, they were detected by deep sequencing in this cohort. M46I/L is considered a major PI mutation and would increase drug resistance levels to PIs along with other mutations. $^{34,42}$ Because no PI drugs were used in the firstline ART in this cohort, these PI mutations were likely natural polymorphisms of HIV-1. The natural polymorphism of M46I has been reported to have a replicative advantage for subtype $\mathrm{B}^{43}$ while the impact of $\mathrm{M} 46 \mathrm{I} / \mathrm{L}$ natural 
polymorphisms on the development of drug resistance in patients is unknown. As Malawi has started to use lopinavir/ ritonavir for second-line regimens ${ }^{44}$ the emergence of DRMs to PIs should be closely monitored.

Evidence is lacking in understanding the real clinical impact of minority DRMs. Clinical trials are needed to accurately evaluate the clinical consequences of these DRMs. However, our results indicate that minority mutations detected by barcoded deep sequencing show an increased or expanded level of resistance to NRTIs and NNRTIs (see mutation scores in Tables 3 and 4). For instance, increased M184IV/I mutations would reduce susceptibility to lamivudine and emtricitabine (scores from 0 to 60 , from susceptible to high-level of resistance). K65R+ $\mathrm{M} 184 \mathrm{~V} / \mathrm{I}$ would reduce susceptibility to tenofovir and didanosine from low-level (scores from 0 to 60 ) to highlevel resistance (scores from 15 to 75). Additionally, other individual thymidine-analog mutations showed an intermediate or high level of resistance to Malawi's firstline regimens (stavudine, lamivudine and nevirapine). These low-frequency mutations detected by the barcoded parallel sequencing added significant values to the resistant reservoir in the HIV-positive population. All mutation data, both majority and minority mutations, can be used by doctors or policy makers as a reference when changing or revising treatment therapy for patients with virologic failure or country first-line regimens in Malawi.

\section{Limitations}

This study had its limitations. First, the sample size was small due to the availability of remnant samples and budget constraints. A statistically-appropriate sample size should be used for population-level estimations of DRMs in order to make a meaningful statement. Second, although significantly increased minority DRMs were observed in the samples collected from pre-ART and patients with virologic failure using Roche 454 barcoded deep sequencing, lack of proper plasmid mutant K65R control in the test might have compromised the accuracy of calculating the K65R mutation rate. Third, some methods in the current study could not be applied further as a result of the 454 platform and technologies being discontinued due to high cost and errors at some homopolymeric regions. However, the designed primers and barcoded strategy in the current study could be applied to other deep sequencing platforms for HIV drug resistance testing or studies.

\section{Conclusion}

In conclusion, our study confirmed that barcoded parallel deep sequencing technology is capable of detecting minority DRMs from clinical patient samples. These minority DRMs not only increased resistance levels to the antiretroviral drugs that are being prescribed, but they also expanded resistance to additional major first-line antiretroviral drugs such as tenofovir. The minority DRMs detected by deep sequencing may be helpful for selecting the optimal regimens for patients initiating ART and for patients who fail first-line regimens.

\section{Acknowledgements}

This work was supported by the President's Emergency Plan for AIDS Relief (PEPFAR) through the United States Centers for Disease Control and Prevention. The findings and conclusions in this paper are those of the authors and do not necessarily represent the official position of the Centers for Disease Control and Prevention. All authors read and approved the final manuscript.

\section{Competing interests}

The authors declare that they have no financial or personal relationships which may have inappropriately influenced them in writing this article.

\section{Sources of support}

None.

\section{Authors' contributions}

C.Y. and Z.Z. conceived and designed the current study. L.A.R. and Z.Z. performed the deep sequencing experiments. K.T. and G.Z. performed deep sequencing analysis and statistical analysis. N.W.-K. and K.M. participated in a prospective cohort study to monitor ART outcomes and drug resistance development in Malawi, including sample collection, data collection and management. J.R.D., N.W. and H.G. performed Sanger sequencing; D.-P.Z., M.F. and S.S. participated in sequencing data collection and management; and J.N. supervised and supported the study. Z.Z. and C.Y. wrote the manuscript.

\section{References}

1. USAID. 17 million people with access to antiretroviral therapy [page on the Internet]. c2016 [cited 2016 Jun 22]. Available from: http://www.who.int/hiv/ mediacentre/news/global-aids-update-2016-news/en/

2. Gunthard HF, Wong JK, Ignacio CC, et al. Comparative performance of highdensity oligonucleotide sequencing and dideoxynucleotide sequencing of HIV type $1 \mathrm{pol}$ from clinical samples. AIDS Res Hum Retroviruses. 1998;14(10):869876. https://doi.org/10.1089/aid.1998.14.869

3. Schuurman R, Brambilla D, de Groot $T$, et al. Underestimation of HIV type 1 drug resistance mutations: Results from the ENVA-2 genotyping proficiency program. AIDS Res Hum Retroviruses. 2002;18(4):243-248. https://doi.org/ program. AIDS Res Hum Retrovi
$10.1089 / 088922202753472801$

4. Palmer S, Kearney M, Maldarelli F, et al. Multiple, linked human immunodeficiency virus type 1 drug resistance mutations in treatment-experienced patients are virus type 1 drug resistance mutations in treatment-experienced patients are
missed by standard genotype analysis. J Clin Microbiol. 2005;43(1):406-413. hissed by standard genotype analysis.

5. Kozal MJ. Drug-resistant human immunodefiency virus. Clin Microbiol Infect. 2009;15(Suppl 1):69-73. https://doi.org/10.1111/j.1469-0691.2008.02687.x

6. Jourdain G, Ngo-Giang-Huong N, Le Coeur S, et al. Intrapartum exposure to nevirapine and subsequent maternal responses to nevirapine-based antiretroviral therapy. N Engl J Med. 2004;351(3):229-240. https://doi.org/10.1056/NEJMoa041305

7. Kapoor A, Kapoor A, Vani SN. Prevention of mother to child transmission of HIV Indian J Pediatr. 2004;71(3):247-251. https://doi.org/10.1007/BF02724278

8. Lecossier D, Shulman NS, Morand-Joubert L, et al. Detection of minority populations of HIV-1 expressing the K103N resistance mutation in patients failing nevirapine. J Acquir Immune Defic Syndr. 2005;38(1):37-42. https://doi. org/10.1097/00126334-200501010-00007

9. Johnson JA, Li JF, Wei X, et al. Minority HIV-1 drug resistance mutations are present in antiretroviral treatment-naive populations and associate with reduced treatment efficacy. PLoS Med. 2008;5(7):e158. https://doi.org/10.1371/journal. pmed.0050158 
10. Simen BB, Simons JF, Hullsiek KH, et al. Terry Beirn Low-abundance drug-resistant viral variants in chronically HIV-infected, antiretroviral treatment-naive patients significantly impact treatment outcomes. J Infect Dis. 2009;199(5):693-701. significantly impact treatment
https://doi.org/10.1086/596736

11. Zhou Z, Wagar N, Devos JR, et al. Optimization of a low cost and broadly sensitive genotyping assay for HIV-1 drug resistance surveillance and monitoring in resource-limited settings. PLoS One. 2011;6(11):e28184. https://doi.org/10.1371/ journal.pone.0028184

12. Brenner B, Routy JP, Quan $Y$, et al. Persistence of multidrug-resistant HIV-1 in primary infection leading to superinfection. AIDS. 2004;18(12):1653-1660. https://doi.org/10.1097/01.aids.0000131377.28694.04

13. Metzner KJ, Bonhoeffer S, Fischer M, et al. Emergence of minor populations of human immunodeficiency virus type 1 carrying the M184V and L90M mutations in subjects undergoing structured treatment interruptions. J Infect Dis. 2003;188(10):1433-1443. https://doi.org/10.1086/379215

14. Hare CB, Mellors J, Krambrink A, et al. Detection of nonnucleoside reverse transcriptase inhibitor-resistant HIV-1 after discontinuation of virologically suppressive antiretroviral therapy. Clin Infect Dis. 2008;47(3):421-424. https:// doi.org/10.1086/589867

15. Martinez-Picado J, Morales-Lopetegi K, Wrin T, et al. Selection of drug-resistant HIV-1 mutants in response to repeated structured treatment interruptions. AIDS 2002;16(6):895-899. https://doi.org/10.1097/00002030-200204120-00009

16. Le T, Chiarella J, Simen BB, et al. Low-abundance HIV drug-resistant viral variants in treatment-experienced persons correlate with historical antiretroviral use. PLoS One. 2009;4(6):e6079. https://doi.org/10.1371/journal.pone.0006079

17. Wang $C$, Mitsuya $Y$, Gharizadeh B, et al. Characterization of mutation spectra with ultra-deep pyrosequencing: Application to HIV-1 drug resistance. Genome Res. 2007;17(8):1195-1201. https://doi.org/10.1101/gr.6468307

18. Hoffmann C, Minkah N, Leipzig J, et al. DNA bar coding and pyrosequencing to identify rare HIV drug resistance mutations. Nucleic Acids Res. 2007;35(13):e91. https://doi.org/10.1093/nar/gkm435

19. Hedskog C, Mild M, Jernberg J, et al. Dynamics of HIV-1 quasispecies during antiviral treatment dissected using ultra-deep pyrosequencing. PLoS One. 2010;5(7):e11345. https://doi.org/10.1371/journal.pone.0011345

20. Dudley DM, Chin EN, Bimber BN, et al. Low-cost ultra-wide genotyping using Roche/454 pyrosequencing for surveillance of HIV drug resistance. PLoS One. 2012;7(5):e36494. https://doi.org/10.1371/journal.pone.0036494

21. Zagordi $\mathrm{O}$, Klein $\mathrm{R}$, Daumer $\mathrm{M}$, et al. Error correction of next-generation sequencing data and reliable estimation of HIV quasispecies. Nucleic Acids Res. 2010;38(21):7400-7409. https://doi.org/10.1093/nar/gkq655

22. Wadonda-Kabondo N, Bennett D, van Oosterhout JJ, et al. Prevalence of HIV drug resistance before and 1 year after treatment initiation in 4 sites in the Malaw antiretroviral treatment program. Clin Infect Dis. 2012;54(Suppl 4):S362-S368. https://doi.org/10.1093/cid/cir987

23. Johnson VA, Calvez V, Gunthard HF, et al. 2011 update of the drug resistance mutations in HIV-1. Top Antivir Med. 2011;19(4):156-164.

24. Bennett DE, Camacho RJ, Otelea D, et al. Drug resistance mutations for surveillance of transmitted HIV-1 drug-resistance: 2009 update. PLoS One. 2009;4(3):e4724. https://doi.org/10.1371/journal.pone.0004724

25. Harrigan PR, Dong W, Wynhoven B, et al. Performance of ReCall basecalling software for high-throughput HIV drug resistance basecalling using "in-house" methods. The XIV international AIDS Conference; 2002 Jul 7-12; Barcelona, Spain. Abstract \#TuPeB4598.

26. WHO. Consolidated guidelines on the use of antiretroviral drugs for treating and preventing HIV infection: Recommendations for a public health approach; 2013; p. 1-272. Geneva, Switzerland: World Health Organization.

27. Zhang G, Cai F, de Rivera IL, et al. Simultaneous detection of major drug resistance mutations of HIV-1 subtype B viruses from dried blood spot specimens by multiplex allele-specific assay. J Clin Microbiol. 2016;54(1):220-222. https://doi. org/10.1128/JCM.02833-15
28. Avidor B, Girshengorn S, Matus N, et al. Evaluation of a benchtop HIV ultradeep pyrosequencing drug resistance assay in the clinical laboratory. I Clin Microbiol. 2013;51(3):880-886. https://doi.org/10.1128/JCM.02652-12

29. Mitsuya $Y$, Varghese $V$, Wang $C$, et al. Minority human immunodeficiency virus type 1 variants in antiretroviral-naive persons with reverse transcriptase codon 215 revertant mutations. J Virol. 2008;82(21):10747-10755. https://doi. org/10.1128/JVI.01827-07

30. Varghese $\mathrm{V}$, Wang E, Babrzadeh F, et al. Nucleic acid template and the risk of a PCR-Induced HIV-1 drug resistance mutation. PLoS One. 2010;5(6):e10992. https://doi.org/10.1371/journal.pone.0010992

31. Larsen BB, Deng W, Maust B, et al. The sensitivity of HIV deep sequencing. 19th Conference on Retroviruses and Opportunistic Infections; Seattle, WA; March 5-8, 2012. Paper \#544.

32. Shafer RW. Low-abundance drug-resistant HIV-1 variants: Finding significance in an era of abundant diagnostic and therapeutic options. J Infect Dis. 2009;199(5):610-612. https://doi.org/10.1086/596737

33. Gianella S, Delport W, Pacold ME, et al. Detection of minority resistance during early HIV-1 infection: Natural variation and spurious detection rather than transmission and evolution of multiple viral variants. J Virol. 2011;85(16):83598367. https://doi.org/10.1128/JVI.02582-10

34. Johnson VA, Brun-Vezinet $F$, Clotet $B$, et al. Update of the drug resistance mutations in HIV-1: Spring 2008. Top HIV Med. 2008;16(1):62-68.

35. Kozal MJ, Chiarella J, St John EP, et al. Prevalence of low-level HIV-1 variants with reverse transcriptase mutation $\mathrm{K} 65 \mathrm{R}$ and the effect of antiretroviral drug exposure on variant levels. Antivir Ther. 2011;16(6):925-929. https://doi.org/10.3851/ IMP1851

36. Bansode V, McCormack GP, Crampin AC, et al. Characterizing the emergence and persistence of drug resistant mutations in HIV-1 subtype $C$ infection using 454 ultra deep pyrosequencing. BMC Infect Dis. 2013;13:52. https://doi. org/10.1186/1471-2334-13-52

37. Coutsinos $\mathrm{D}$, Invernizzi $\mathrm{CF}, \mathrm{Xu} \mathrm{H}$, et al. Template usage is responsible for the preferential acquisition of the K65R reverse transcriptase mutation in subtype $C$ variants of human immunodeficiency virus type 1. J Virol. 2009;83(4):2029-2033. https://doi.org/10.1128/JVI.01349-08

38. Keulen W, Nijhuis M, Schuurman R, et al. Reverse transcriptase fidelity and HIV-1 variation. Science. 1997:275(5297):229; author reply 230-221. https://doi. org/10.1128/JVI.74.14.6262-6268.2000

39. Frost SDW, Nijhuis M, Schuurman R, et al. Evolution of lamivudine resistance in human immunodeficiency virus type 1-infected individuals: The relative roles of drift and selection. J Virol. 2000;74(14):6262-6268.

40. Qari SH, Winters $M$, Vandamme AM, et al. A rapid phenotypic assay for detecting multiple nucleoside analogue reverse transcriptase inhibitor resistant HIV-1 in plasma. Antivir Ther. 2002;7(2):131-139. https://doi.org/ 10.1155/2012/637263

41. Jiao L, Li H, Li L, et al. Impact of novel resistance profiles in HIV-1 reverse transcriptase on phenotypic resistance to NVP. AIDS Res Treat. 2012;2012:637263.

42. Nwobegahay JM, Bessong PO, Masebe TM, et al. Prevalence of antiretroviral drug resistance mutations and HIV-I subtypes among newly-diagnosed drug-naive persons visiting a voluntary testing and counselling centre in northeastern South Africa. J Health Popul Nutr. 2011;29(4):303-309. https://doi.org/10.3329/jhpn. v29i4.8444

43. Manosuthi W, Thongyen S, Nilkamhang S, et al. HIV-1 drug resistance-associated mutations among antiretroviral-naive Thai patients with chronic HIV-1 infection. $J$ Med Virol. 2013;85(2):194-199. https://doi.org/10.1002/jmv.23452

44. Hosseinipour MC, van Oosterhout JJ, Weigel R, et al. The public health approach to identify antiretroviral therapy failure: High-level nucleoside reverse transcriptase inhibitor resistance among Malawians failing first-line antiretrovira therapy. AIDS. 2009;23(9):1127-1134. https://doi.org/10.1097/QAD.0b013e3283 2ac34e 